\title{
Towards an understanding of the interactions of Trypanosoma cruzi and Trypanosoma rangeli within the reduviid insect host Rhodnius prolixus
}

\author{
PATRÍCIA AZAMBUJA ${ }^{1}$, NORMAN A. RATCLIFFE ${ }^{2}$ and ELOI S. GARCIA ${ }^{1}$ \\ ${ }^{1}$ Department of Biochemistry and Molecular Biology, Instituto Oswaldo Cruz, Fundação Oswaldo Cruz \\ Av. Brasil 4365, 21045-900 Rio de Janeiro, RJ, Brasil \\ ${ }^{2}$ Biomedical and Physiologial Research Group, School of Biological Sciences, University of Wales \\ Swansea, Singleton Park, Swansea, SA28PP, United Kingdom \\ Manuscript received on March 3, 2005; accepted for publication on March 30, 2005; \\ contributed by ELOI S. GARCIA*
}

\begin{abstract}
This review outlines aspects on the developmental stages of Trypanosoma cruzi and Trypanosoma rangeli in the invertebrate host, Rhodnius prolixus. Special attention is given to the interactions of these parasites with gut and hemolymph molecules and the effects of the organization of midgut epithelial cells on the parasite development. The vector insect's permissiveness to $T$. cruzi, which develops in the vector gut, largely depends on the host nutritional state, the parasite strain and the molecular interactions with trypanolytic compounds, lectins and resident bacteria in the gut. T. rangeli invades the hemocoel and once in the hemolymph, can be recognized and activates the defense system of its insect vector, i.e., the prophenoloxidase system, phagocytosis, hemocyte microaggregation, superoxide and nitric oxide activity and the eicosanoid biosynthesis pathway. Taken together, these findings not only provide a better understanding of the interactions parasite - insect vector, but also offer new insights into basic physiological processes involved in the parasites transmission.
\end{abstract}

Key words: Trypanosomes, Rhodnius prolixus, vector, parasites.

\section{INTRODUCTION}

The co-evolution of parasites and insects has promoted the development of a powerful and sophisticated strategy based on both physiological and biochemical vector and/or parasite mechanisms, which act to facilitate parasite development or its disruption in the invertebrate host. The debate concerning

Dedicated to the memory of Prof. Dr. Herman Lent by initiative of Pedro L. Oliveira, Instituto de Bioquímica Médica. Universidade Federal do Rio de Janeiro (UFRJ), RJ, Brasil.

* Member Academia Brasileira de Ciências

Correspondence to: Dr. Patrícia Azambuja

E-mail: azambuja@ioc.fiocruz.br the Trypanosoma cruzi and T. rangeli- triatomine vector interactions results from their complexity and modes of parasite transmission (Garcia and Azambuja 1991, Kollien and Schaub 2000). Like T. cruzi, T. rangeli infects humans and a wide range of other mammals and has a similar geographic distribution (Guhl and Vallejo 1998).

Many factors are believed to contribute to the establishment of trypanosomes in the gut of the invertebrate host. However, differences in the biological cycles between both parasites exist: T. rangeli, but not $T$. cruzi, invades the hemocoel and physicochemical barriers such as the gut membranes, which 
are important to T. cruzi development, but not to $T$. rangeli in the digestive tract (Gonzalez et al. 1999, Azambuja and Garcia, personal observation).

This review outlines research on the developmental aspects of Trypanosoma cruzi and Trypanosoma rangeli in the insect vector, which may reveal new perspectives for the control of Chagas disease.

\section{TRYPANOSOMA CRUZI AND VECTOR INTERACTIONS}

Before considering more details of the vector - parasite interactions, it is important to understand some aspects of the parasite life cycle in the invertebrate host. Points of contact between T. cruzi, the etiologic agent of Chagas disease, and its triatomine vectors begin with ingestion of the infective blood meal. Blood feeding is essential for the parasite growth in all instars and in the adult insect (Kollien and Schaub 1998). Interactions between T. cruzi and its vector insects begin with the arrival of an infected blood meal in the insect gut. During feeding, the trypomastigotes forms from the blood of the infected vertebrate host are ingested by the insect (Garcia and Azambuja 1991, Kollien and Schaub 2000). After a few days in the stomach (anterior part of the midgut) of the insect most of the bloodstream trypomastigotes transform into epimastigotes and some spheromastigotes (Garcia and Azambuja 1991, Kollien and Schaub 2000). Subsequently, mainly in the intestine (posterior part of the midgut), the epimastigotes divide repeatedly by binary division and can attach to the perimicrovillar membranes in the intestinal cells (Gonzalez et al. 1999). At later stages in the rectum, a proportion of the epimastigotes attach to the rectal cuticle and transform into metacyclic trypomastigotes that are eliminated with the feces and urine and are able to infect the vertebrate host (Garcia and Azambuja 1991, Kollien and Schaub 2000).

Since the gut of triatomines is the first environment for the establishment of T. cruzi infection, we studied the possible influence of digestive enzymes on parasite development (Garcia 1987), developed suitable bioassays for specific aspects of triatomine physiology and biochemistry in the gut (for review see Garcia and Azambuja 1997), and analyzed a variety of factors, such as the clone used and feeding with azadirachtin, influencing the life cycle of $T$. cruzi in the gut of the vector, $R$. prolixus (Garcia and Azambuja 1991).

Results showed that feeding $R$. prolixus with the inhibitor of acid SH-proteinase, pepstatin, had no effect on rates of $T$. cruzi infection (Garcia and Gilliam 1980). Also, trypomastigotes from singlecell-isolates of two clones of $T$. cruzi produced different rates of growth because of different population doubling times of the parasites and only one clone underwent epimastigote to metacyclic trypomastigote transformation (Garcia and Dvorak 1982). Results therefore indicate that not only the kinetics of $T$. cruzi epimastigote multiplication but also the transformation to metacyclic trypomastigotes depend on the strains and clones of the infecting parasite (Garcia et al. 1984b). In addition, the passage of homogeneous strains of $T$. cruzi through the invertebrate host gut was identical with the host was unable to modify any biological, biochemical or genetic characteristics of the parasite (Garcia et al. 1986b).

Although many factors are thought to be important to the establishment of T. cruzi infection in the gut of the vector, only a few molecules have been implicated, such as a stomach lytic factor (Azambuja et al. 1983, 1989), lectins (Pereira et al. 1981, Mello et al. 1996) and hemoglobin fragments (Garcia et al. 1995), all of which have been tested directly against the parasite. The work of Pereira et al. (1981) reported lectins in the digestive tube and hemolymph of $R$. prolixus and suggested that these molecules could be involved in the development of $T$. cruzi in the triatomine bug. Mello et al. (1996) demonstrated differences in the development of three strains of $T$. cruzi in the gut of $R$. prolixus and related the infectivity of these to the ability of the digestive tube extract to agglutinate these parasitesin vitro. Interestingly, lectins agglutinated the Dm28c clone of $T$. cruzi which achieved high infectivity levels while, in contrast, the Y strain of the parasite which was not agglutinated, was lysed and failed to develop in the vector gut (Azambuja et al. 
1989, Mello et al. 1996, 1999).

Frainderaich et al. (1993) demonstrated that metacyclogenesis of $T$. cruzi is promoted in vitro by an $\alpha^{\mathrm{D}}$-globin-derived peptide present in hemoglobin corresponding to residue 1-40 from the amino terminus found in the gut of Triatoma infestans. Synthetic peptides having the amino terminal globin sequences and containing conserved domains spanning amino acid residues 30 to 40 are recognized by a surface receptor in epimastigote cells and stimulate $T$. cruzi adenylyl cyclase (Frainderaich et al. (1993). Garcia et al. (1995) also studied in vivo in $R$. prolixus, the effects of hemoglobin and synthetic peptides carrying $\alpha^{\mathrm{D}}$-globin fragments on both the growth and transformation of $T$. cruzi epimastigotes into metacyclic trypomastigotes. This differentiation in the insect gut occurred when hemoglobin and synthetic peptides corresponding to residues 3049 and 35-73 of the $\alpha^{\mathrm{D}}$-globin were added to the plasma diet. However, synthetic peptide 41-73 did not induce differentiation of epimastigotes even in the presence of the two former peptides so that peptide 41-73 appeared to block the action of these stimulatory peptides. In addition, the whole hemoglobin molecule was shown to be a very important blood component for the growth of parasites (Garcia et al. 1995). These data identified an unusual molecular mechanism, which modulates the dynamics of transformation of epimastigotes into metacyclic trypomastigotes in the triatomine vectors gut.

Recently, Azambuja et al. (2004) opened an exciting new research area by studying the effects of resident bacteria in the stomach of $R$. prolixus on erythrocyte lysis and T. cruzi infection. Following feeding, bacteria rapidly multiplied and the number of surviving Y strain of $T$. cruzi in the stomach declined drastically, while infection with Dm28c clone remained stable. Hemolytic bacteria were isolated and identified as Serratia marcescens biotype A1a (referenced as RPH), which produces the pigment, prodigiosin. In vitro experiments, comparing incubation of RPH or $S$. marcescens SM365, a prodigiosin pigment producer, or $S$. marcescens DB11, a none pigmented variant, as a control, with erythro- cytes and T. cruzi demonstrated that: (i) at $0^{\circ} \mathrm{C}$ or $30^{\circ} \mathrm{C}$, both SM365 and RPH reduced the populations of Y strain, but not of the DM28c clone, and DB11 was unable to lyse either T. cruzi strain; and (ii) all three strains of $S$. marcescens were able to lyse erythrocytes (Azambuja et al. 2004). These results suggest that $S$. marcescens trypanolytic activity from the SM365 and RPH strains is distinct from the hemolytic activity and that prodigiosin is an important factor for the trypanolytic action of the bacteria in the gut of the vector. The study of bacteria in the gut of invertebrate hosts may be important and provide new tools to block the development of parasites in the insect vector.

One of the more interesting outcomes of the T. cruzi - vector interactions resulted from the investigations made with the compound azadirachtin, a natural growth inhibitor from the neem tree ( $\mathrm{Aza}$ dirachta indica A. Juss), which strongly interferes with the neuroendocrine regulation of the insect hormone titers (Garcia and Rembold 1984, Garcia et al. 1984a, 1986a, 1990). This compound not only affects the development of triatomines but also the establishment of T. cruzi infection in the gut of different species of triatomines (Garcia et al. 1989, Gonzalez and Garcia 1992, Gonzalez et al. 1999, Kollien et al. 1998).

Nogueira et al. (1997) analyzed the process of interaction of epimastigotes and trypomastigotes of T. cruzi (Dm 28c clone and Y strain) with the intestinal epithelium of $R$. prolixus in vitro. Their observations showed that both parasite developmental stages move towards the epithelial surface. However, while trypomastigotes did not attach to the epithelial surface, all epimastigotes strongly attached through the flagellum, preferentially at the periphery of the cells. When the $R$. prolixus larvae were decapitated or treated with azadirachtin, the T. cruzi epimastigotes were unable to adhere to the stomach or intestinal epithelium (Gonzalez et al. 1999). This may result from the effect of these treatments on factors present in the perimicrovillar membrane, which interact with glycoinositol phospholipids (GIPLs) molecules abundant on the epimastigote plasma 
membrane and involved in the process of parasite insect host-interaction (Garcia and Azambuja, unpublished data). Thus, manipulation of the physiological condition of the vector host may affect the development of $T$. cruzi, with decapitation, head transplantation, azadirachtin and ecdysone therapy consequently all influencing the parasite development. Insects that received these treatments showed a distinct effect characterized by ultrastructural disorganization of the midgut epithelial cells of $R$. prolixus and indicated that the prothoracicotropic hormone (PTTH)-ecdysone pathway interferes with T. cruzi survival and development in its vectors (Gonzalez et al. 1999). These results provide the first clear evidence showing the importance of the insect endocrine system in establishing T. cruzi infection in the vector.

\section{TRYPANOSOMA RANGELI AND VECTOR INTERACTIONS}

T. rangeli, is a parasite generally considered nonpathogenic to humans and animals but can be infective to triatomines (Watkins 1971). Little is known about the life cycle of $T$. rangeli in the vertebrate hosts, although infections in humans and wild animals have been reported (Guhl and Vallejo 1998). After ingestion as trypomastigotes, $T$. rangeli differentiates into epimastigotes and multiplies in the gut of its insect vector (usually Rhodnius prolixus) and commonly invades the hemolymph to continue its growth, and complete its development in the salivary glands, where metacyclogenesis takes place (Watkins 1971, Hecker et al. 1990, Garcia et al. 1994, Mello et al. 1995). Interestingly, insects with salivary glands infections pierce the host skin more frequently draw less blood and at a slower rate than controls when feeding on mammals (Garcia et al. 1994). Additionally, infected salivary glands significantly reduced levels of both apirase activity and reactive nitrogen groups. It seems that salivary gland infection of $T$. rangeli manipulates the vector ability to locate blood vessels by affecting the salivary antihemostatic properties, thus enhancing the possibility of intradermal inoculation of parasites into the mammalian host (Garcia et al. 1994).

Other important compounds involved in the parasite-vector interactions are lectins. These compounds not only block carbohydrates exposed on the parasite surface of but also inhibit the attachment of the $T$. rangeli in both midgut (Oliveira and de Souza 1997) and salivary glands (Basseri et al. 2003). After $T$. rangeli invasion of the hemolymph from the midgut, the parasites are taken up by the $R$. prolixus hemocytes and divide therein before escaping and invading the salivary glands (Tobie 1970). It is not clear if the invasion of parasites in the hemocytes is mediated solely by phagocytosis or by active invasion by the parasite in a parasitophorous vacuole. The latter hypothesis was observed by transmission electron microscopy studies, which showed that plasmatocytes were able to ingest epimastigote forms of the parasite, which were then found within a parasitophorous vacuole (de Oliveira and de Souza 2003).

Once in the hemolymph, $T$. rangeli can be recognized and activates the defense system of its insect vector. Several aspects of the humoral and cellular immune responses have been investigated in T. rangeli - triatomine interactions, including lysozymes and trypanolytic activity (Mello et al. 1995, Gomes et al. 1999), prophenoloxidase (proPO) activation (Mello et al. 1995, Gomes et al. 1999, 2003, Garcia et al. 2004a), phagocytosis and hemocyte microagglutination (Mello et al. 1995, Garcia et al. 2004a,b), hemolymph agglutination (Mello et al. 1996), and superoxide and nitric oxide generation (Whitten et al. 2001).

Studies carried out on the activation of the proPO system of $R$. prolixus infected with short and long epimastigote forms of $T$. rangeli demonstrated that short, but not long, epimastigotes activated the formation of melanin (Gomes et al. 1999). Whitten et al. (2001) also studied the immune response of $R$. prolixus in vivo following the inoculation of two strains and two developmental forms of $T$. rangeli. The results showed that after $24 \mathrm{hr}$ the H14 strain, which failed to multiply and invade the salivary glands, stimulated significantly higher levels of 
prophenoloxidase, superoxide and nitrites than the Choachi strain, which rapidly multiplied in the hemolymph to invade the salivary glands for transmission to the vertebrate host. Usually, short form epimastigotes stimulated greater superoxide and proPO responses than long epimastigotes in both parasite strains. In addition, when the NADPH oxidase inhibitor, N-ethylmaleimide, or the inducible nitric oxide synthase inhibitor, S-methyl isothiourea sulfamide, are injected into groups of $R$. prolixus they caused significantly higher insect mortalities after inoculation with parasites of either strain compared with those of uninfected control insects (Whitten et al. 2001). This indicates that both NADPH oxidase and nitric oxide synthase activity may be involved in the immune response of $R$. prolixus infection by $T$. rangeli. In Rhodnius prolixus, NOS gene expression and nitrite levels are modulated in the stomach, midgut and fat body following in vivo challenge with $T$. cruzi or $T$. rangeli. Elevations in nitrite activity in the gut wall occur during the response to infection by both species of trypanosomes but are particular high with $T$. cruzi, which could explain why this parasite fails to cross the midgut wall into the hemocele (Whitten et al., unpublished data).

In many insects species, cellular immune responses to pathogens depend on two kinds of reactions: (i) nodulation and encapsulation and (ii) phagocytosis by hemocytes. Nodule formation involves hemocyte degranulation and activation of the proPO system, attachment of the developing nodule to host tissues and the beginning of phagocytic activity by the plasmatocytes (Ratcliffe and Rowley 1979).

It was shown that in $R$. prolixus hemocyte monolayers, both $T$. cruzi and $T$. rangeli are capable of inducing hemocyte/parasite clump formation. A purified galactoside-binding lectin markedly enhanced the activation of clump formation by $T$. rangeli in $R$. prolixus hemocyte monolayers, with an increase in clump size and hemocyte aggregation (Mello et al. 1999). Takle (1988) and Mello et al. (1995) described the aggregation process, following injection of $T$. rangeli and T. cruzi into R. prolixus. The purified hemolymph lectin also significantly reduced the motility and survival of $T$. rangeli culture short forms, but not the long forms, when they were incubated in vitro (Mello et al. 1999). In normal infections, a few days after inoculation of $T$. rangeli short forms into the hemolymph of the insect, they disappear to be replaced by a massive colonization by long forms (Mello et al. 1995) and it may be that the hemolymph lectin mediates this process. There is little evidence for melanization of the hemocyte microaggregation, despite Alvarenga et al. (1990) having described melanized nodules in response to abiotic or biological challenges. Apparently, the $T$. rangeli long forms suppress the proPO system that produces melanin (Gomes et al. 1999, 2003).

Recently, our laboratory investigated the effects of eicosanoid biosynthesis inhibitors on hemocyte microaggregation and proPO-activating system in the hemolymph, and on parasitemia and mortality of $R$. prolixus infected with $T$. rangeli. Hemocoelic injection of $T$. rangeli epimastigotes into insects that were previously fed on blood containing an inhibitor of phospholipase A2, dexamethasone, a specific inhibitor of the cyclooxygenase pathway, indomethacin, and a non-selective lipoxygenase inhibitor, NDGA, reduced hemocyte aggregation, attenuated the proPO system in the hemolymph and enhanced parasitemia and mortality induced by the parasite challenge (Garcia et al. 2004a). These authors demonstrated that the effects obtained by dexamethasone administered orally were counteracted by inoculation of the insects with arachidonic acid. It seems that Rhodnius' immune response to a parasite infection is modulated by a physiological system that includes eicosanoid biosynthesis pathways. Garcia et al. (2004b) also examined the effect of feeding $R$. prolixus larvae on blood containing $T$. rangeli on the cellular immune system, focusing on aggregation reactions in the hemolymph when the insect is subsequently challenged by inoculation with the same parasite. This is an interesting model since the double infections (oral and hemocoelic inoculation) are similar to what occurs in nature. They 
showed a reduced number of hemocyte aggregations in the hemolymph, enhanced numbers of parasites in the hemolymph as well as increased mortality in these insects. All these effects were counteracted by the combined inoculation of $R$. prolixus with $T$. rangeli together with arachidonic acid (Garcia et al. 2004b). They concluded that oral infection with T. rangeli inhibits the release of arachidonic acid, which is a mediator of hemocyte aggregation reactions in the hemolymph of $R$. prolixus infected with T. rangeli (Garcia et al. 2004a,b).

\section{CONCLUSIONS}

The above mini review indicates the complexity of the trypanosome - triatomine relationship and indicates that research into basic aspects of parasite - invertebrate host interactions can reveal subtle mechanisms for the establishment of parasite infection, that are transmitted by blood-feeding insect vectors. Regardless of the medical importance of triatomines and their high suitability for experimentation, remarkably little is understood about their interactions with trypanosomes. The demand for this kind of study is increasing daily with the need to find new methods of vector control. Many points remain to be answered, such as: (i) what other molecules are present in the digestive tract and involved as modulators of parasite infection by controlling the interaction with the gut epithelial cells and perimicrovillar membranes whose functions are complex and poorly understood; (ii) how do the eicosanoid pathways and hormones induce immune depression in the vector at the molecular level; (iii) how do genes, parasites and vector molecules interact and impact the superoxide and nitric oxide and other reactive radicals; and (iv) what, if any, role do the inducible antimicrobial peptides play as they could potentially influence survival and parasite establishment in the host vector.

\section{ACKNOWLEDGMENTS}

This work was supported by grants from Conselho Nacional de Desenvolvimento Científico e Tecno- lógico (CNPq), Fundação Oswaldo Cruz (Papes) and Volkswagen Foundation. PA and ESG (CNPq) and NAR (Faperj) are Research Fellows.

\section{RESUMO}

Esta revisão destaca aspectos dos estágios do desenvolvimento de Trypanosoma cruzi $\mathrm{e}$ Trypanosoma rangeli $\mathrm{em}$ seu hospedeiro invertebrado, Rhodnius prolixus Atenção especial é dada às interações desses parasitas com moléculas presentes no tubo digestivo e na hemolinfa e efeitos da organização das células epiteliais sobre o desenvolvimento do parasita. A susceptibilidade do inseto vetor ao T. cruzi, o qual desenvolve no tubo digestivo, dependem do estado nutricional do hospedeiro, da cepa do parasita e de interações moleculares com compostos tripanolíti$\cos$, lectinas e bactérias residentes no tubo digestivo. O $T$. rangeli invade a hemocele e pode ser reconhecido e ativar o sistema de defesa do inseto vetor, ou seja, o sistema profenoloxidase, fagocitose, microagregação hemocitária, atividade de superóxido e óxido nítrico e a via de biossíntese dos eicosanóides. Estes fatos fornecem uma compreensão melhor das interações parasita-inseto vetor, e também oferecem novas idéias sobre os processos básicos envolvidos na transmissão parasitária.

Palavras-chave: Tripanosomas, Rhodnius prolixus, vetor, parasitas.

\section{REFERENCES}

Alvarenga NJ, Bronfen E, Alvarenga RJ and BarRACO MA. 1990. Triatomine's hemocytes and granuloma formation and biological and non-biological materials. Mem Inst Oswaldo Cruz 85: 377-379.

Azambuja P, Guimarães JA and Garcia ES. 1983. Hemolytic factor from the stomach of Rhodnius prolixus: evidence and partial characterization. J Insect Physiol 29: 833-837.

Azambuja P, Mello CB, D'Escoffier LN and GarCIA ES. 1989. In vitro cytotoxicity of Rhodnius prolixus hemolytic factor and mellitin towards different trypanosomatids. Brazilian J Med Biol Res 22: 597-599.

Azambuja P, Feder D and Garcia ES. 2004. Isolation of Serratia marcescens in the midgut of Rhodnius prolixus: Impact on the establishment of the parasite, Trypanosoma cruzi, in the vector. Exp Parasitol 107: 89-96. 
BASSERI HR, Tew IF AND RATClifFe NA. 2003. Identification and distribution of carbohydrate moieties on the salivary glands of Rhodnius prolixus and their possible involvement in attachment/invasion by Trypanosoma rangeli. Exp Parasitol 100: 226-234.

DE Oliveira MA And de Souza W. 2003. Further morphological studies on the behavior of Trypanosoma rangeli in the hemocytes of Rhodnius prolixus. Parasitol Int 52: 299-307.

Frainderaich D, Pena C, Isola EL, Lammel EM, Coso O, Diazanel A, Pongor S, Baralle F, Torres HN AND Flawiá MM. 1993. Stimulation of Trypanosoma cruzi adenyl cyclase by na $\alpha^{\mathrm{D}}$-globon fragment from Triatoma hindgut. Proc Nat Acad Sci USA 90: 10140-10144.

GarcIa ES. 1987. The digestion of Triatominae. In: Brenner RR and Stoka A (Eds), Chagas' Disease Vector II. Anatomic and Physiological Aspects, Vol. II, CRC Press, Florida, p. 47-59.

Garcia ES and Azambuja P. 1991. Development and interactions of Trypanosoma cruzi within the insect vector. Parasitol Today 7: 240- 244.

Garcia ES and Azambuja P. 1997. Infection of triatomines with Trypanosoma cruzi. In: CRAMPTON JM et al. (Eds), Molecular Biology of Insect Disease Vectors: A Methods Manual. Chapman and Hall, p. 146-155.

Garcia ES AND Dvorak JA. 1982. Growth and development of two Trypanosoma cruzi clones in the arthropod Dipetalogaster maximus. Am J Trop Med Hyg 31: 259-262.

Garcia ES and Gilliam FC. 1980. Trypanosoma cruzi development is independent of protein digestion in the gut of Rhodnius prolixus. J Parasitol 66: 1052-1053.

Garcia ES and Rembold H. 1984. Effects of azadirachtin on ecdysis of Rhodnius prolixus. J Insect Physiol 30: 939-941.

Garcia ES, Azambuja P, Forster H and Rembold H. 1984a. Feeding and molt inhibition by azadirachtins A, B, and 7-acethyl-azadirachtin A in Rhodnius prolixus nymphs. Z Naturforsch 39: 1155-1158.

Garcia ES, Vieira E, Gomes JEPL and Gonçalves AM. 1984b. Molecular biology of the interaction Trypanosoma cruzi/invertebrate host. Mem Inst Oswaldo Cruz 29: 33-37.
Garcia ES, Uhl M and Rembold H. 1986a. Azadirachtin, a chemical probe for the study of molting processes in Rhodnius prolixus. Z Naturforsch 41: 711-715.

Garcia ES, Vieira E, Gonçalves AM, Morel CM, Alves MJ And Colli W. 1986b. A strain of Trypanosoma cruzi, and its biochemical characterization after passage through different invertebrate hosts. Ann Trop Med Parasitol 80: 361-363.

Garcia ES, Gonzalez MS, Azambuja P and RemBOLD H. 1989. Chagas' disease and its insect vector. Effects of azadirachtin A on the interaction of a triatomine host (Rhodnius prolixus) and its parasite (Trypanosoma cruzi). Z Naturforch 44: 317-322.

Garcia ES, Luz N, Azambuja P and Rembold H. 1990. Azadirachtin depresses the release of prothoracicotropic hormone in Rhodnius prolixus larvae: evidence from head transplantations. J Insect Physiol 36: 679-682.

Garcia ES, Mello CB, Azambuja P and Ribeiro JMC. 1994. Rhodnius prolixus: salivary anti-hemostatic components decrease with Trypanosoma rangeli infection. Exp Parasitol 78: 287-293.

Garcia ES, Gonzalez MS, Azambuja P, Baralle FE, Frainderaich D, Torres HN and Flawiá MM. 1995. Induction of Trypanosoma cruzi metacyclogenesis in the hematophagous insect vector by hemoglobin and peptides carrying globin sequences. Exp Parasitol 81: 255-261.

Garcia ES, Machado EMM and Azambuja P. 2004a. Effects of eicosanoid biosynthesis inhibitors on the prophenoloxidase-activating system and microaggregation reactions in the hemolymph of Rhodnius prolixus infected with Trypanosoma rangeli. J Insect Physiol 50: 157-165.

Garcia ES, Machado EMM and Azambuja P. 2004b. Inhibition of hemocyte microaggregation reactions in Rhodnius prolixus larvae orally infected with Trypanosoma rangeli. Exp Parasitol 107: 31-38.

Gomes SAO, Feder D, Thomas NE, Garcia ES AND Azambuja P. 1999. Rhodnius prolixus infected with Trypanosoma rangeli: in vivo and in vitro experiments. J Invertebr Pathol 73: 289-293.

Gomes SAO, Feder D, Garcia ES and Azambuja P. 2003. Suppression of the prophenoloxidase system in Rhodnius prolixus orally infected with Trypanosoma rangeli. J Insect Physiol 49: 829-837. 
GonZalez MS AND GaRCia ES. 1992. Effect of azadirachtin on the development of Trypanosoma cruzi in different species of triatomine insect vectors: longterm and comparative studies. J Invertebr Pathol 60: 201-205.

Gonzalez MS, Nogueira NF, Mello CB, de Souza W, Schaub GA, Azambuja P and Garcia ES. 1999. Influence of brain and azadirachtin on Trypanosoma cruzi development in the vector, Rhodnius prolixus. Exp Parasitol 92: 100-108.

Guhl F and Vallejo A. 1998. Trypanosoma (Herpetosoma) rangeli Tejera, 1920 - an updated review. Mem Inst Oswaldo Cruz 98: 435-442.

Hecker H, Schwarzenbach M and Rudin W. 1990. Development and interactions of Trypanosoma rangeli in and with the reduviid bug, Rhodnius prolixus. Parasitol Res 76: 311-318.

Kollien AH and Schaub GA. 1998. The development of Trypanosoma cruzi (Trypanosomatidae) in the reduviid bug Triatoma infestans (Insect): Influence of starvation. J Euk Microbiol 45: 59-63.

Kollien AH and Schaub GA. 2000. The development of Trypanosoma cruzi in Triatominae. Parasitol Today 16: 381-387.

Kollien AH, Gonçalves TC, Azambuja P, Garcia ES AND Schaub GA. 1998. The effect of azadirachtin on fresh isolates of Trypanosoma cruzi in different species of triatomines. Parasitol Res 84: 286-290.

Mello CB, Garcia ES, Ratcliffe NA and AzamBUJA P. 1995. Trypanosoma cruzi and Trypanosoma rangeli: interplay with hemolymph components of Rhodnius prolixus. J Invertebr Pathol 65: 261-268.

Mello CB, Azambuja P, Garcia ES and Ratcliffe NA. 1996. Differential in vitro and in vivo behaviour of three strains of Trypanosoma cruzi in the gut and hemolymph of Rhodnius prolixus. Exp Parasitol 82: 112- 268 .
Mello CB, Nigan Y, Garcia ES, Azambuja P, NewTON RP AND RATClifFe NA. 1999. Studies of a hemolymph lectin isolated from Rhodnius prolixus and its interaction with Trypanosoma rangeli. Exp Parasitol 91: 289-296.

Nogueira NFS, Gonçalves MS, Garcia ES AND DE SouzA W. 1997. Effect of azadirachtin A on the fine structure of the midgut of Rhodnius prolixus. J Invertebr Pathol 69: 58-63.

Oliveira MA AND DE Souza W. 1997. Interaction of Trypanosoma rangeli with midgut cells of Rhodnius prolixus treated with lectins. Mem Inst Oswaldo Cruz.

Pereira MEA, Andrade AFB And Ribeiro JMC. 1981. Lectins of distinct specificity in Rhodnius prolixus interact selectively with Trypanosoma cruzi. Science 211: 597-600.

RatCliffe NA AND Rowley AF. 1979. Role of hemocytes in defence against biological agents. In: GUPTA AP (Ed), Insect Hemocytes Development, Forms, Functions, and Techniques. Cambridge University Press, Cambridge, UK, p. 331-414.

TAKLE GB. 1988. Studies on the cellular immune response of insects toward the insect pathogen Trypanosoma rangeli. J Invertebr Pathol 51: 64-72.

ToBIE EJ. 1970. Observations on the development of Trypanosoma rangeli in the hemocoel of Rhodnius prolixus. J Invertebr Pathol 16: 118-125.

WATKINS R. 1971. Histology of Rhodnius prolixus infected with Trypanosoma rangeli. J Invertebr Pathol 17: 59-66.

Whitten MM, Mello CB, Gomes SA, Nigan Y, AzambUja P, Garcia ES AND RATCLIFFE NA. 2001. Role of superoxide and reactive nitrogen intermediates in Rhodnius prolixus (Reduviidae)/ Trypanosoma rangeli interactions. Exp Parasitol 98: 44-57. 